\title{
UV Photodiodes Response to Non-Normal, Non-Colimated and Diffusive Sources of Irradiance
}

\author{
María-Paz Zorzano, Javier Martín-Soler and Javier Gómez-Elvira \\ Centro de Astrobiología (INTA-CSIC), Carretera de Ajalvir km 4 \\ Torrejón de Ardoz, Madrid, \\ Spain
}

\section{Introduction}

A photodiode is a type of photodetector capable of converting incident light into either current or voltage, depending upon the mode of operation. The substrate used to make the photodiode sensing part is critical to defining its properties. In particular, only photons with sufficient energy to excite electrons across the material's bandgap will induce photocurrents. Materials commonly used to produce photodiodes include: Silicon (for the wavelength range 190-1100 nm), Germanium (for 400-1700 nm), Indium-Gallium arsenide (for 800-2600 $\mathrm{nm}$ ) and Lead (II) sulphide (1000-3500 nm), among others. In many applications the semiconductor diode is placed within an opaque housing with different possible sizes (such as TO18, TO5, TO3..) and hermetically sealed with a cover of a material that allows transmission of the desired wavelength range of observation and permits radiation to reach the sensitive part of the device. Some applications require measurement of one specific wavelength range and it is essential that other radiation wavelengths of the range where the substrate shows responsivity do not contribute to the photodiode's current. For this purpose a filter is mounted above the detector to select the wavelength range of responsivity. Common configurations of filtered photodiodes include a metallic platform to hold the filter at an intermediate height between the cover window and the detector, see Figure 1. This mechanical configuration allows the implementation of different filters preserving the external packing dimensions and physical properties of the photodiode sealing with independence of the filter type.

The geometry of arrangement of these elements (cover, housing, and sensing device) defines the nominal field of view (FOV) or geometrical FOV of the sensor. A careful look at this configuration shows that, because of secondary internal reflections against the inner walls of the sensor housing, the sensor may also be excited by photons from trajectories with incident angles greater than this nominal geometrical FOV. Notice that the filter crystal is thick, flat and cut in square shape. Its implementation on top of the sensing dice covers the nominal FOV but leaves a fraction of the photodiode horizontal cross-section uncovered, see Figure 2. As we will explain later, this will have implications for non-normal, noncollimated and diffusive irradiance sources. 

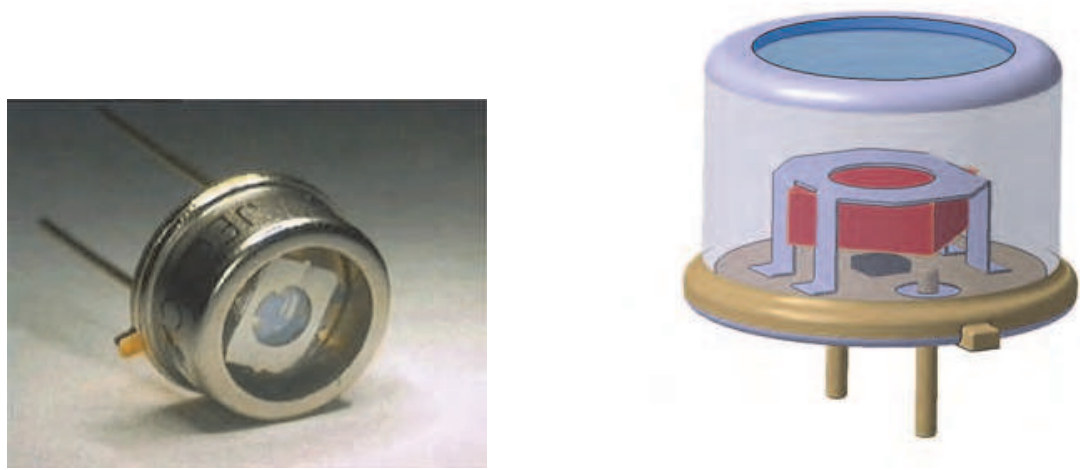

Fig. 1. (Left) Commercial hermetically sealed filtered ultraviolet (UV) photodiode with TO5 housing and the filter mounted on a metallic platform. (Right) Geometrical configuration of the photodiode. The filter crystal is here represented in red and the $\mathrm{SiC}$ detector dice in black. The filter is mounted on the metallic structure but does not reach the side of the housing.

Because of changes in the refractive index as the light beam crosses the boundary between two media there is an angle variation which is given by Snell law. The light ray bends toward the normal when the light enters a medium of greater refractive index, and away from the normal when entering a medium of lesser refractive index. For this later case there is a limiting angle beyond which all the rays should be reflected that is called the critical angle of incidence. For instance, in the case of a transition from a quartz crystal to gaseous nitrogen or to air the nominal critical angle is roughly $40^{\circ}$. In theory, for incident angles greater than this angle the light rays should be totally reflected by the cover crystal. However in most cases there is a range of angles where a fraction is reflected and a fraction transmitted. In addition in some implementations, such as the one analyzed here, the covering window is slightly curved. This induces additional angular distortions for nonnormal incidence and in practice shifts the critical angle of incidence towards greater values. In summary for the case considered here, beyond the nominal FOV there is a wider angle of allowed incoming rays, what we will here call the critical angle FOV, where incident rays are allowed to pass inside the photodiode housing. Photon rays contained between the nominal FOV and the critical angle FOV may get inside the photodiode housing, be reflected in the walls, avoid the filter and be trapped between the filter and the sensing substrate dice. These photons may thus excite the dice and contribute to the total photodiode output current avoiding the filter selective bandpass action and producing an unexpected current leak. This is schematically represented in Figure 2.

In summary, for a given arbitrary configuration where the radiation from a complex radiating environment excites a photodiode, part of the radiation may be reflected by the cover window and part may pass with slightly different exit angle, part may be contained within the FOV and traverse the filter and part may bounce within the inner part of the sensor housing and hit the dice with a new incidence angle (and here again it may be absorbed, or reflected). When these effects are present the response of the dice to the incident irradiance is neither the one tabulated in the device specifications by the manufacturer (which is usually defined for normal incidence) nor the one obtained in 
standard laboratory calibrations. For any practical photodiode use, where the incident light is non-normal and non-collimated, these deviations should be explored under representative operation conditions.

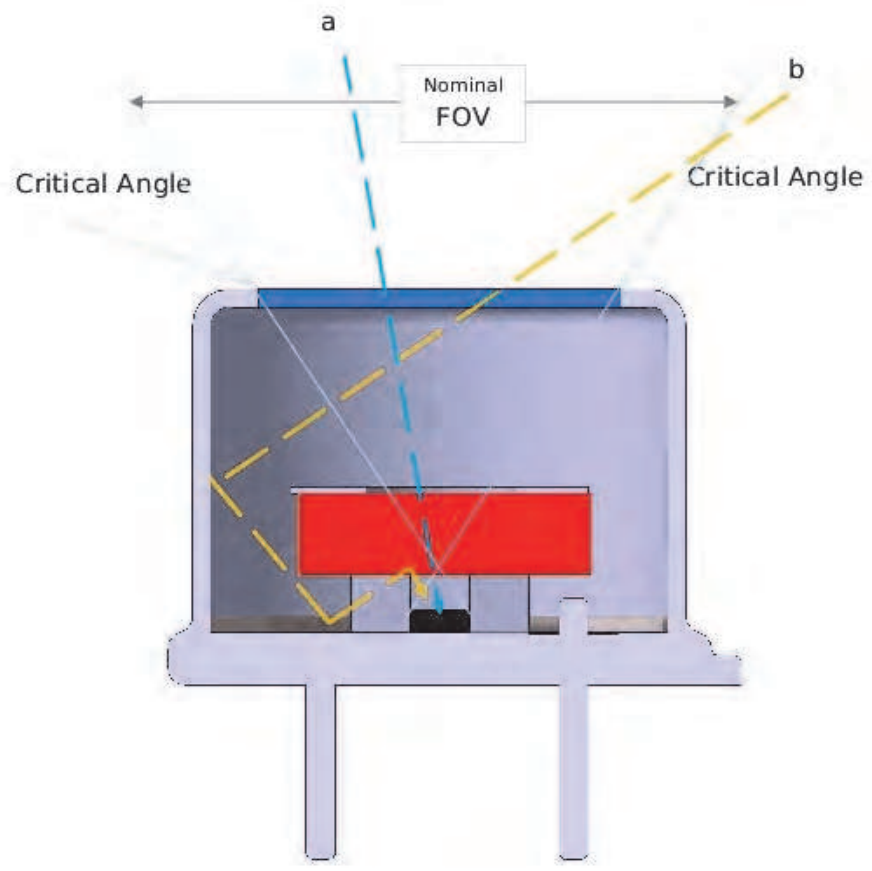

Fig. 2. Nominal field of view (FOV) and critical angle conceptual representation: All ray beams, such as (a), within the FOV solid angle hit the sensing dice after traversing the filter. Photon rays that traverse the window cover with angles of incidence such as (b) hit the inner housing walls and reach the sensing dice after multiple reflections, avoiding the filter selective action.

It is the aim of this chapter to illustrate these effects experimentally with a specific study case and to describe an experimental setup that can be used to quantify the relevance of each contribution. In particular, we describe here the calibration and operation studies of the (ultraviolet) UV photodiodes of the UV sensor for REMS (Rover Environmental Monitoring Station) instrument on-board the MSL rover to Mars (NASA 2012) [Gómez-Elvira et al., 2008]. This sensor consists of a set of 6 photodiodes with different responsivity spectral ranges. One of the photodiodes has no filter and is sensitive in the total $\mathrm{SiC}$ responsivity range (from here on named $\mathrm{ABC}$ ). The other 5 photodiodes correspond to filtered bands named A,B,C,D and E, see Figure 4. Each broadband measurement provides a crude evaluation of the incident irradiance in its relevant spectral range: photodiode $C$ provides a first order estimate of the level of biologically damaging irradiance; photodiodes A and B provide an estimate that may be comparable with terrestrial irradiances while photodiode ABC gives an estimate of the total UV irradiance, and photodiodes $\mathrm{D}$ and $\mathrm{E}$ are designed to match two UV channels of the MARCI instrument, on-board the Mars Reconnaissance 
Orbiter (MRO) satellite to enable direct comparison with reflectance measurements from the top of the atmosphere. The chosen UV photodiodes have a nominal FOV of $\pm 30^{\circ}$. This is the solid angle contained between the normal to the dice and the imaginary line connecting the sensing dice and the opaque border of the top of the caging, as shown in Figure 2. The photodiodes are mounted in a circular pattern within a metallic box on the rover deck, facing the sky. Each one is embedded within a magnet (to deflect the trajectories of in-falling magnetic dust and protect the window from Martian dust deposition, mimicking the effect of the magnet experiment of the MER rovers) [Kinch et al. 2006]. A scheme of this setup with the nominal field of view of $\pm 30^{\circ}$ of a photodiode is shown in Figure 3. The whole REMSUV setup, in an anodized aluminium box of $55 \mathrm{~mm} \times 68 \mathrm{~mm} \times 16 \mathrm{~mm}$ with a D25 connector, weights only $72 \mathrm{~g}$. Photodiodes have the advantage of being small in size, light and robust for operation under harsh conditions such as those expected for the MSL rover.

This sensor will deliver for the first time in-situ surface ultraviolet irradiance measurements that will provide ground-truth to radiative transfer models and satellite reflectance measurements as well as first order estimates of biological and chemical doses and UV opacities. A solid understanding of the UV radiation behaviour of the Martian atmosphere is important for photochemical models of the atmosphere [Rodrigo et al., 1990], for the chemistry of the surface minerals [Holland 1978, Mukhin et al., 1996, Quin et al. 2001], has biological implications and is paramount for the assessment of the possible habitability of the Martian surface [Cockell et al. 2000, Patel et al. 2002, Patel et al. 2003, Patel et al. 2004a, Patel et al. 2004B, Cordoba-Jabonero et al. 2003, Cordoba-Jabonero et al. 2005]. In addition, satellite [Mateshvili et al. 2006, Montmessin et al. 2006] and Martian-ground based measurements of UV radiation [Zorzano and Córdoba-Jabonero 2007], are important to retrieve, through radiative transfer studies, [Zorzano et al. 2005], accurate information on atmosphere aerosols, in particular aerosol size distribution, load, dust and cloud dynamics. It is also relevant to estimate the ozone content which in turn serves as a proxy for Mars atmospheric water vapour.

Previous space missions designed to explore the UV Martian conditions, such as the failed Beagle lander mission, also considered a similar UV sensor concept based on photodiodes [Patel et al. 2002]. However, because of their simplicity they also have certain limitations. This study summarizes the evaluation of the response of this setup to representative operation conditions.

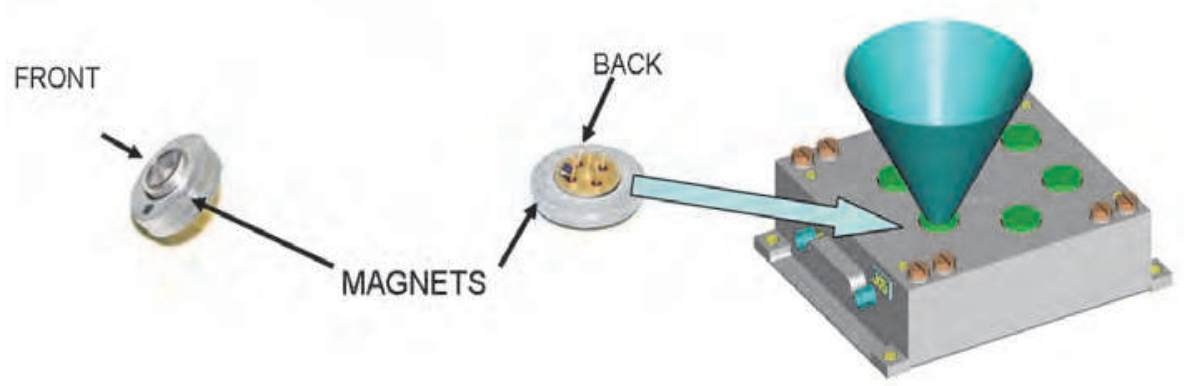

Fig. 3. (Left) Front view of the photodiode and magnet. (Centre) Back view of the photodiode with pins and magnet. (Right) Schematic representation of the nominal FOV of the photodiode within REMS-UV box. 
In this space application, the direct radiation source is the Sun. The incident UV radiation comes both as a direct beam, with incident direction according to the solar zenithal angle at the moment of observation, and as diffuse UV irradiance. This diffuse component is the product of the scattering interaction between the incident solar radiation with the dust aerosols and molecules of the thin Martian atmosphere [Zorzano et al. 2005, Zorzano and Córdoba-Jabonero 2007, Zorzano et al. 2009]. The response of the photodiodes in this operation environment shall be investigated.

In summary, we have three scenarios to consider. When the photon ray is within the geometrical FOV, the direct beam is expected to be filtered. When the direct beam is between the geometrical FOV and the critical angle FOV, secondary reflections against the wall may allow extra photons to reach the dice avoiding the filter and thus inducing a current leakage produced by an unfiltered contribution. Finally we shall consider the response to the background diffuse irradiance, i.e. the radiation that has suffered scattering with the atmosphere and reaches the sensor window from almost any direction. In this case the sensor is excited by the diffuse irradiance contained within the solid angle of FOV, which is a significant fraction of the sky diffused irradiance, and shall be filtered. The extra diffuse radiation coming from rays with angles greater than this FOV, but still within the critical angle $\mathrm{FOV}$, can also excite the $\mathrm{SiC}$ dice through secondary reflections and, for some photodetectors such as the one considered here, avoiding the filter action. The fraction of diffuse radiation that gets to the dice not being filtered is proportional to the difference between the nominal FOV and the critical angle FOV. If the downwelling diffuse irradiance is uniform then this is a pure geometrical factor. There are second order corrections to this due to the specific reflective, absorption and transmission characteristics of each filter that will be also experimentally observed.

\section{Characterization of the response under laboratory conditions}

\subsection{Spectral calibration of the response with a direct collimated beam of normal or inclined incidence}

The response of the sensor to a direct beam of collimated light can be calibrated under controlled operation conditions. This has been done to characterize the spectral responsivity of each photodiode and its dependence with angle of incidence. Its dependence with temperature, the linearity of the response, the degradation with aging and thermal cycling were also analyzed for this specific application in space instrumentation but the results of these tests are beyond the scope of this chapter and shall not be discussed here.

A UV source, focusing optics, a monochromator, a calibrated beam splitter, a detector and a multimeter have been used to calibrate spectrally the response of the photodiodes. One of the photodiodes was sent to The National Physical Laboratory (NPL) (UK's National Measurement Institute) for reference calibration and the results of this calibration setup were referenced with this measurement. The spectral responsivity of each of the 6 chosen photodiodes to a collimated direct beam at normal incidence and ambient temperature is shown in Figure 4. The spectral response of the $\mathrm{SiC}$ sensing dice is the one labelled as $\mathrm{ABC}$, namely the one of the photodiode without filter. The rest of photodiodes have a filter and the spectral responsivity is the result of the combined effect of this filter with the underlying $\mathrm{SiC}$ responsivity.

The same study has been performed for inclined incidence. Figure 5 shows the measured decay of responsivity (as percentage with respect to the one at normal incidence) for the 
peak wavelength (the wavelength of maximal responsivity) for all chosen photodiodes together with a 7 th order polynomial fit (the r.m.s. error of the fit is $5,3 \%$ ). We observe in this graph both the departure from cosine law and the response of the dices to radiation for incident angles beyond the nominal FOV. This study has been performed for different polar angles and the result is qualitatively similar (not shown), concluding that the response of the photodiode to a direct collimated beam depends mostly on the azimuth angle. At the edge of the nominal FOV $\left( \pm 30^{\circ}\right)$ the response has decayed to a $40 \%$ with respect to the normal (and not to $85 \%$ as would be expected by a pure cosine like response). Beyond this point there is still significant signal (a $20 \%$ of the maximal). The difference between the slow decay of $\mathrm{ABC}, \mathrm{A}$ and $\mathrm{E}$ and the quick decay of $\mathrm{B}, \mathrm{C}$, and $\mathrm{D}$ is because the maximum of the spectral response of the later ones shows a shift of about $20 \mathrm{~nm}$ towards lower wavelength ranges. Furthermore, it is clearly observed in this graph that all the photodiodes show significant responsivity beyond the nominal FOV of $\pm 30^{\circ}$.

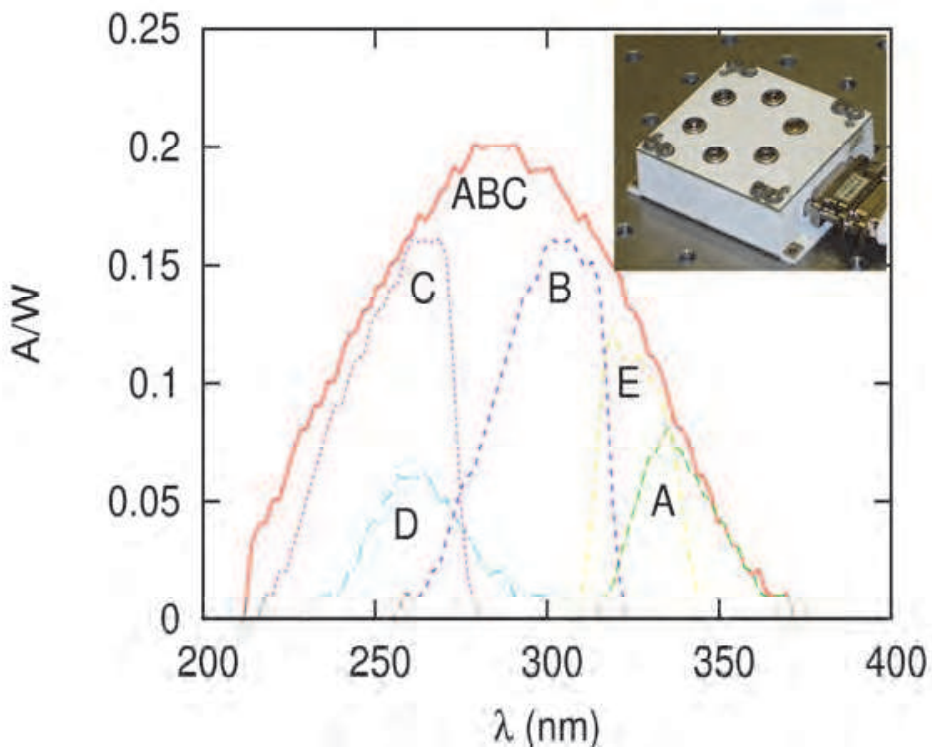

Fig. 4. (Insert) View of the REMS UV box with 6 photodiodes. (Graph) Spectral responsivity, calibrated at ambient temperature with a collimated beam at normal incidence.

\subsection{Spectral characterization of the response of the non-filtered contribution with a direct collimated beam at normal incidence}

To evaluate qualitatively the spectral weight of the unfiltered contribution, a photodiode was manipulated to separate, in the total current signal, the contribution from the filtered signal and the unfiltered contribution. The same setup used for the spectral calibration of flight model units was used here.

A C type photodiode was opened (by cutting the TO5 housing); an opaque element (a small aluminium plate) was placed on top of the filter, blocking the passage of light rays through this path. Photodiodes that have suffered this manipulation are here named " $o b$ ". These photodiodes deliver a current only when photons hit the SiC sensing dice avoiding the filter 
(which is in this case blocked). By doing this we evaluate the unfiltered contribution of a direct beam, i.e. the direct beam photons that reach and excite the sensing dice avoiding the nominal FOV and thus avoiding the filter. The photodiode base was glued again to the can. Notice that after this manipulation the TO5 cage is filled in by ambient air instead of the original nitrogen encapsulation.

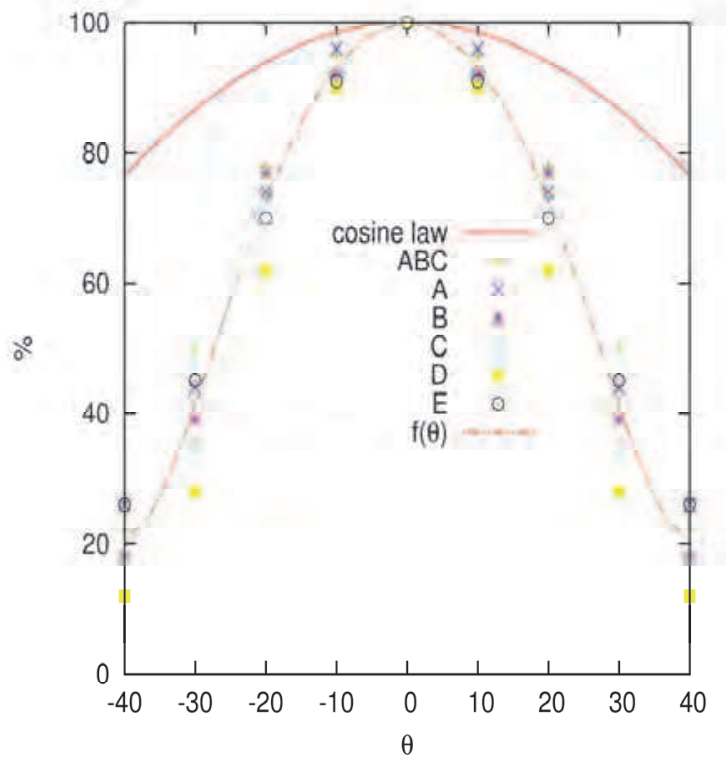

Fig. 5. Zenithal angle dependence of the responsivity to an inclined collimated beam for the $\mathrm{SiC}$ unfiltered photodiode (ABC) and 5 filtered photodiodes (A, B, C, D and E). Comparison of the angle dependence between cosine-like decay and a polynomial fit of the measured data with angle. Notice that for a collimated source the photodiodes show significant responsivity beyond the nominal FOV of $\pm 30^{\circ}$.

In Figure 6 we show the spectral response of this open-blocked photodiode and compare it with the one of a normal C photodiode. Be aware that the filter partially transmits, partially reflects, and partially absorbs. Each specific filter has a different behaviour with respect to transmission and absorption, tuned to select the transmissions properties only. When photons are trapped below the filter, bouncing back and forth until they hit the dice, the filter may also absorb a spectral part of the irradiance. In this specific example a relevant part of the signal of the UVA range seems to be partially blocked, but most of the UVB range radiation is hitting the $\mathrm{SiC}$ dice through secondary wall reflections creating a current leak. Thus we may expect different responses for blocked photodiodes of different filters. As we will see later this is indeed observed.

\subsection{Characterization of the angular response of the non-filtered contribution with a divergent beam}

To qualitatively evaluate under laboratory conditions the angular response of the unfiltered contribution, a new photodiode was manipulated to separate, in the total current signal, the 
contribution from the filtered signal and the unfiltered contribution. This time a D type photodiode was opened and blocked.

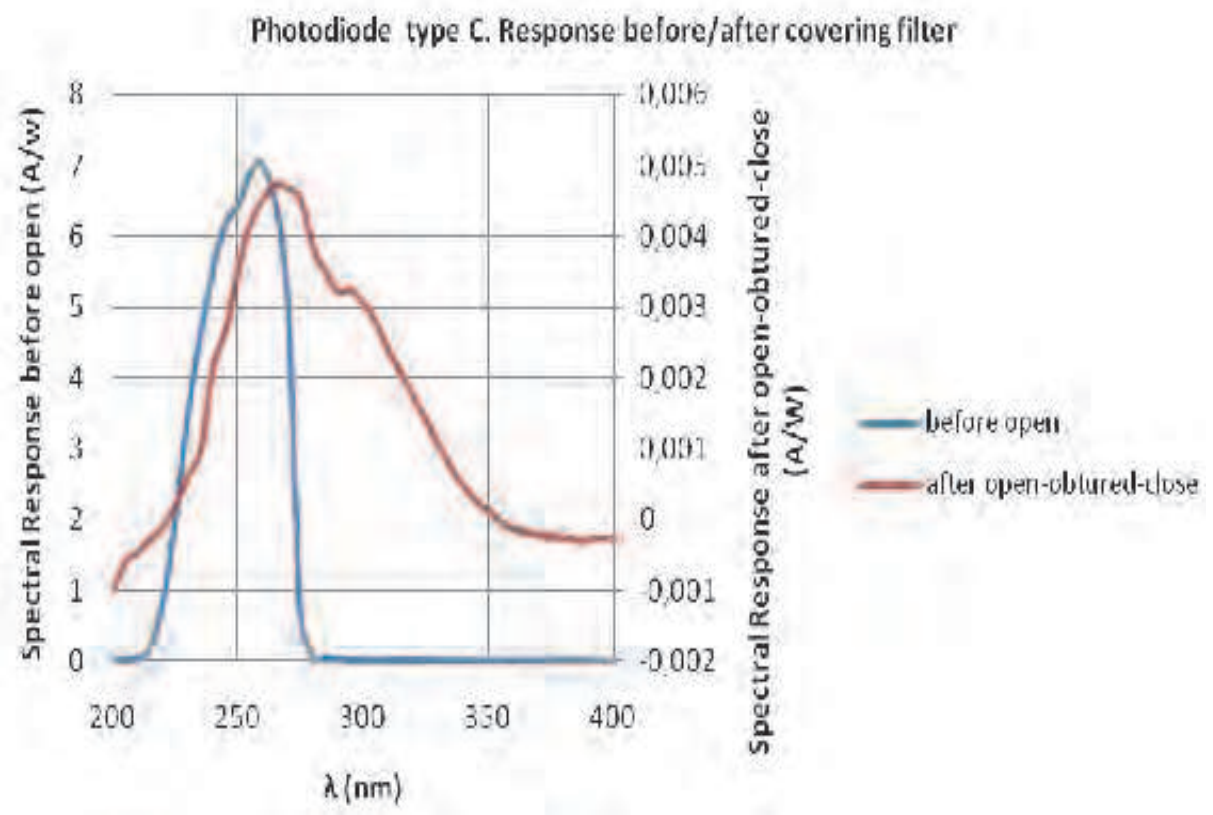

Fig. 6. Spectral response of the open-blocked C photodiode and comparison with original nominal spectral response. The open-blocked responsivity shows the spectral signature of the non-filtered contribution in a $\mathrm{C}$ photodiode and extends beyond the nominal UVC range.

A xenon (Xe) light source was placed at a distance of $24 \mathrm{~cm}$ to the photodiode (such that the cone of collimated light diverges) and its inclination angle with respect to the diode was varied, see Figure 7. This setup was hard to control but the results give significant qualitative information. The maximal unfiltered response is at normal incidence, when the divergent beam hits the photodiode from above. A fraction of the divergent light beam intersects the housing walls; the light in these rays is internally reflected and reaches the dice. As the source goes to lower azimuthal positions, i.e. greater zenithal angle, the signal is reduced up to an angle of roughly $15-20^{\circ}$ where it has a minimum. At this point the light beam is half-way to the nominal field of view limit $\left(30^{\circ}\right)$ which is given by the mechanical obstruction of the upper edge in the housing wall. From this point on most of the direct rays hit the side walls. They are then scattered and reflected and reach the dice avoiding the filter. The signal increases then up to roughly $40^{\circ}-45^{\circ}$. Beyond this point there is a rapid decay and light is partially reflected back at the crystal interface because of the critical angle limit, see Figures 7 and 8 . In view of this, we conclude that for field campaign observations, when the Sun direct light beam has a zenithal angle close to $40^{\circ}-45^{\circ}$ (or equivalently an altitude angle between $50^{\circ}-45^{\circ}$ ), the measurements of photodiodes where the unfiltered contribution may be comparable to the filtered one shall be discarded. 


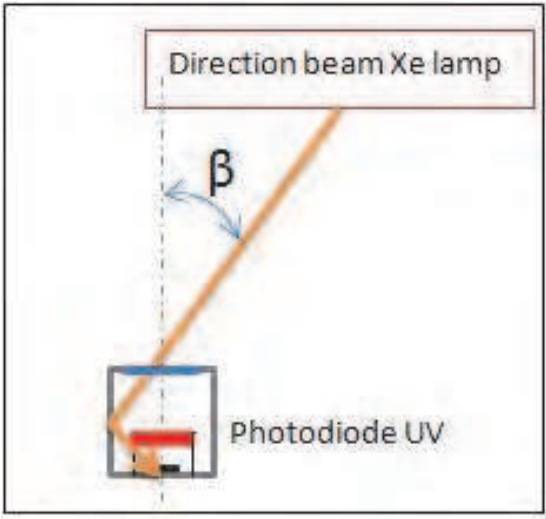

Fig. 7. Schematic representation of the laboratory setup. The beam of the Xe lamp was inclined with respect to the norm to the photodiode. The D photodiode was open, its filter was blocked, and the sensor housing was glued again, to quantify the angular dependence of the non-filtered contribution.

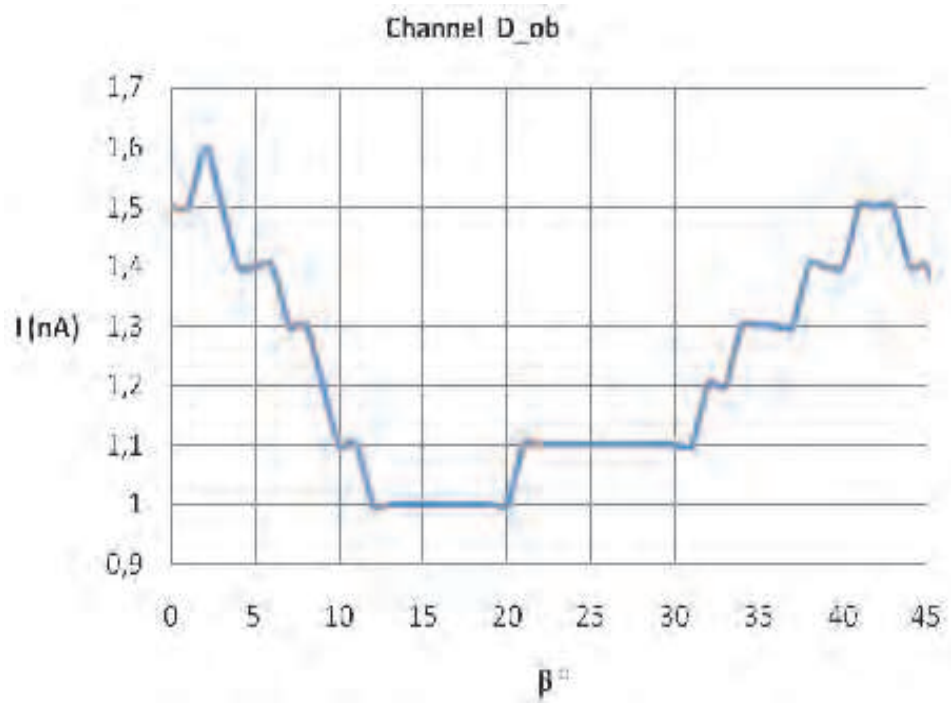

Fig. 8. Non-filtered photodiode's zenithal angle response with an inclined divergent light source exciting a blocked $\mathrm{D}$ type photodiode. There is a maximum in the induced current at normal incidence, when a fraction of the cone of the divergent beam hits the inner walls of the caging. As the beam is inclined there is a secondary maximum at $40^{\circ}-45^{\circ}$, when the centre of the direct divergent beam hits the walls, and is reflected downwards to the sensing dice (avoiding the blocked filter).

Through these experiments we have illustrated the existence of internal reflections for direct beam sources. We therefore redefine a REMS-UV operational strategy that discards observations when the Sun direct beam is in the vicinity of $40-45^{\circ}$ w.r.t. the norm. We will 
evaluate how relevant this internal reflection contribution is for the unavoidable and continuous background of diffusive irradiance.

\section{Response characterization under representative operation conditions}

To investigate the response to an extended distant source such as the Sun with a background source of diffuse irradiance a special implementation was prepared to operate outdoors in a clear sky day, see Figure 9. For Terrestrial atmospheric measurements, we expect channels C and $\mathrm{D}$ to be especially sensitive to the non-filtered contribution because their filters are centred on the ozone absorption band and therefore on Earth the signal in these channels shall be negligible. Any extra signal must be due to an unfiltered contribution.

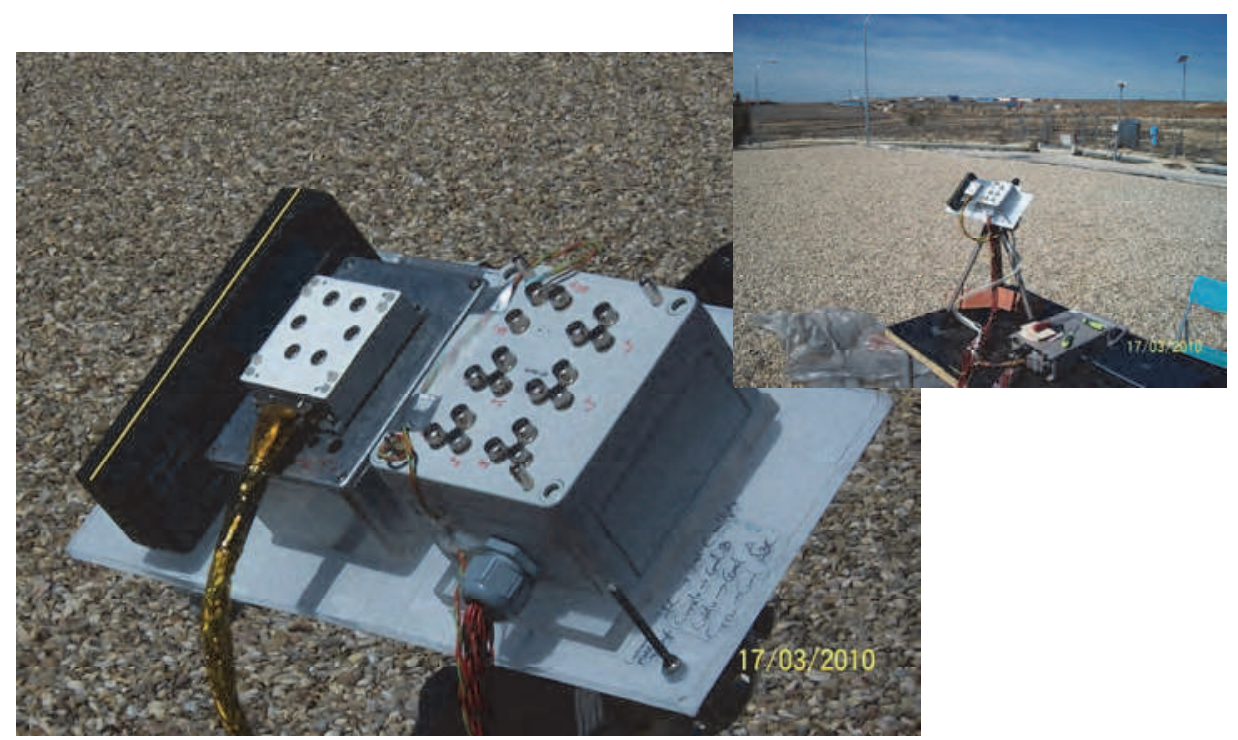

Fig. 9. Engineering model for field campaign measurements. The direct beam (Sun) angle of incidence can be varied by adjusting the platform inclination.

Three different photodiodes types were used per channel: a flight model unit, this one shall be here named $f m$, and two engineering photodiodes that are manipulated. In these last two photodiodes, because of the manipulation (loss of encapsulated nitrogen, mechanical distortion and misalignment after cutting and gluing the housing) we may expect a variation in the response with respect to the flight model units. One of these photodiodes, which is simply opened and glued again, is used as control reference to discard the influence of the manipulation alone (namely loss of the encapsulated gas), this one is from here on named op. The other one is opened, its filter is blocked, and then it is glued again, this one shall be named $o b$.

\subsection{Angular characterization of the unfiltered diffuse radiation}

The photodiode platform was placed horizontally, facing the sky. During this day the maximal Solar Zenithal Angle (SZA) was at $57.5^{\circ}$, and therefore the trajectory was very low 
with respect to the FOV of the photodiodes. The recorded signal was thus the response to the sky diffuse irradiance contained within the solid angle of view of the photodiode. This includes both the diffuse irradiance within the nominal FOV (filtered contribution) and the rest, up to the critical angle FOV (unfiltered contribution). The measured current varies smoothly as the SZA changes along the day. For photodiode $\mathrm{C}$, in the vicinity of the maximal SZA, the diffuse unfiltered contribution $(o b)$ is about $30 \%$ of the total signal in the control photodiode (op) whereas for big SZA the unfiltered signal is about $50 \%$ of the total one, see Figure 10. This is a smooth function and may be easily interpolated. The fm response is shown for reference.

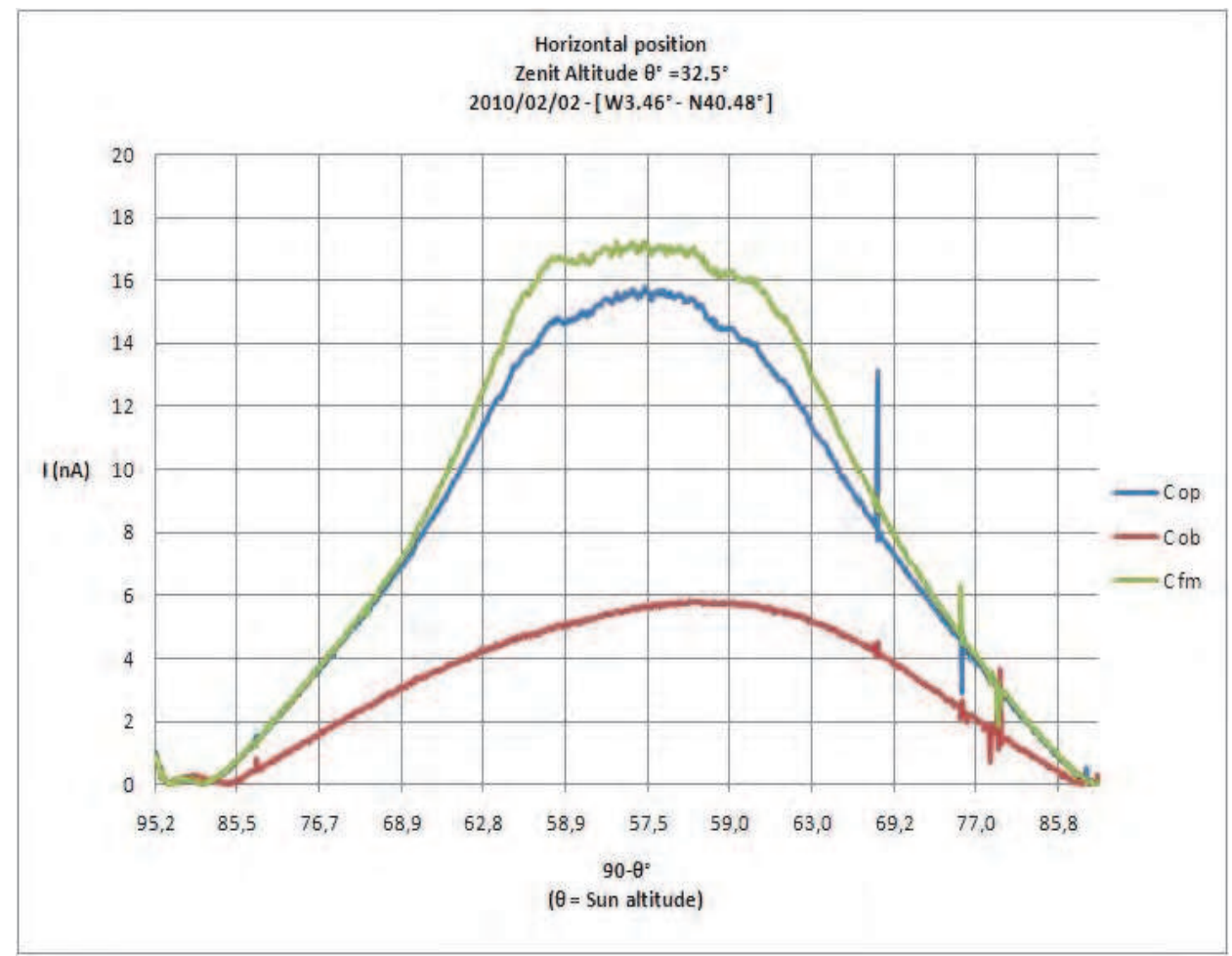

Fig. 10. Comparison of the evolution of the three $C$ photodiodes: flight-model $(\mathrm{fm})$, openblocked $(o b)$ and C open (op) measurement along the day, as the Sun traverses the sky. For this configuration the sun transit avoids both the nominal FOV and the critical angle FOV. Thus the measured current is produced in response to the diffuse irradiance alone.

In Figure 11 we compare this measurement for different $o b$ channels. Since their filters are blocked their response is basically associated to the response of the SiC dice. When the filter is blocked, the order of magnitude of the signal of different blocked photodiodes is similar but there are differences of the order of up to $30 \%$ among them (which may be due to geometrical factors after manipulation or to differences in the coating of the lower part of filters). The averaged intensity of this current is about 50 times less the one of the unfiltered photodiode ABC, see Figure 11. 


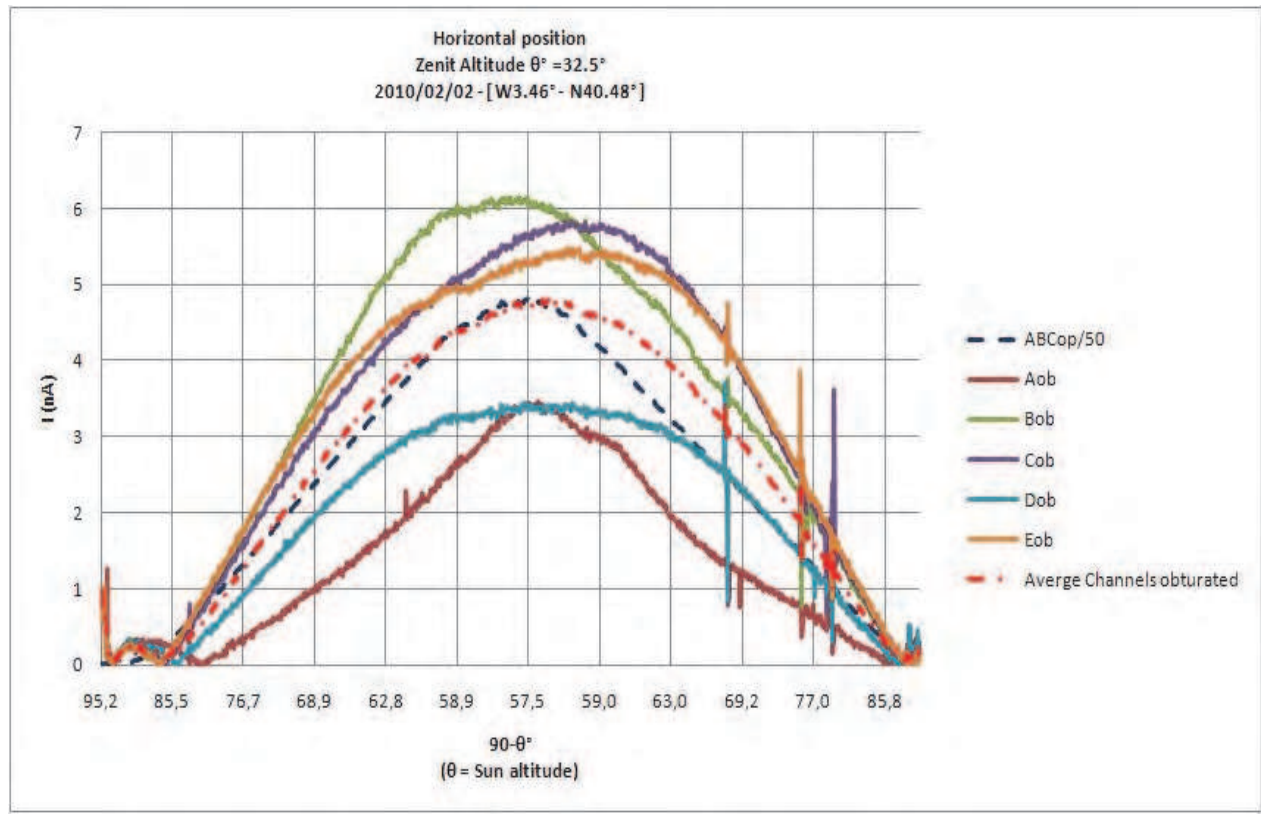

Fig. 11. Comparison of the unfiltered response of all photodiodes, averaged unfiltered response and rescaled (by 50) current of the total $A B C$ photodiode in a configuration where only diffuse irradiance reaches the photodiode.

This suggests that, for this kind of photodiode, a fraction of the UV sky diffuse irradiance avoids the central filter of the photodiode and hits the bottom $\mathrm{SiC}$ diode directly producing a leakage signal of the order of about $2 \%$ of the total $U V$ ( $A B C$ channel) induced current. If the channel of observation expects a very small signal for its nominal spectral range, this current leakage can be comparable or even greater than the one of interest. In particular, as shown above for the $\mathrm{C}$ channel, this represents almost $30 \%$ of the total measured current of the nominal channel, or even $50 \%$ for big SZA.

\subsection{Angular characterization of the unfiltered direct beam contribution}

For the next experimental setup the sensors are again facing the sky, pointing upwards. If the Sun during its trajectory is never within the nominal FOV of the photodiode but passes within the critical angle FOV there are two observable maxima at $45^{\circ}$ that can be seen in the measurements of all photodiodes (both manipulated and original). See Figure 12 for an example of the response of the $C$ photodiode when the solar transit never enters its nominal FOV. The two maxima at $45^{\circ}$ correspond to direct beam wall reflections and thus appear also as unfiltered contribution in the $o b$ photodiode. In this setup the photodiodes collect both the inner wall reflection of the direct beam rays (from the Sun) and the diffuse radiation rays (from the sky). The direct beam induced maxima are at about $45^{\circ}$. Beyond this point, when the Sun leaves the critical angle FOV, we should be able to obtain information about the envelope of the diffuse signal as in the example above. Indeed beyond $50^{\circ}$ there is a change in the shape of the angle dependent response, at this moment the direct beam is fully reflected because of critical angle issues and the current is induced only by the 
background sky diffuse irradiance (the one studied above). Also in this case we observe that for big SZA the nonfiltered signal is about $50 \%$ of the total one.

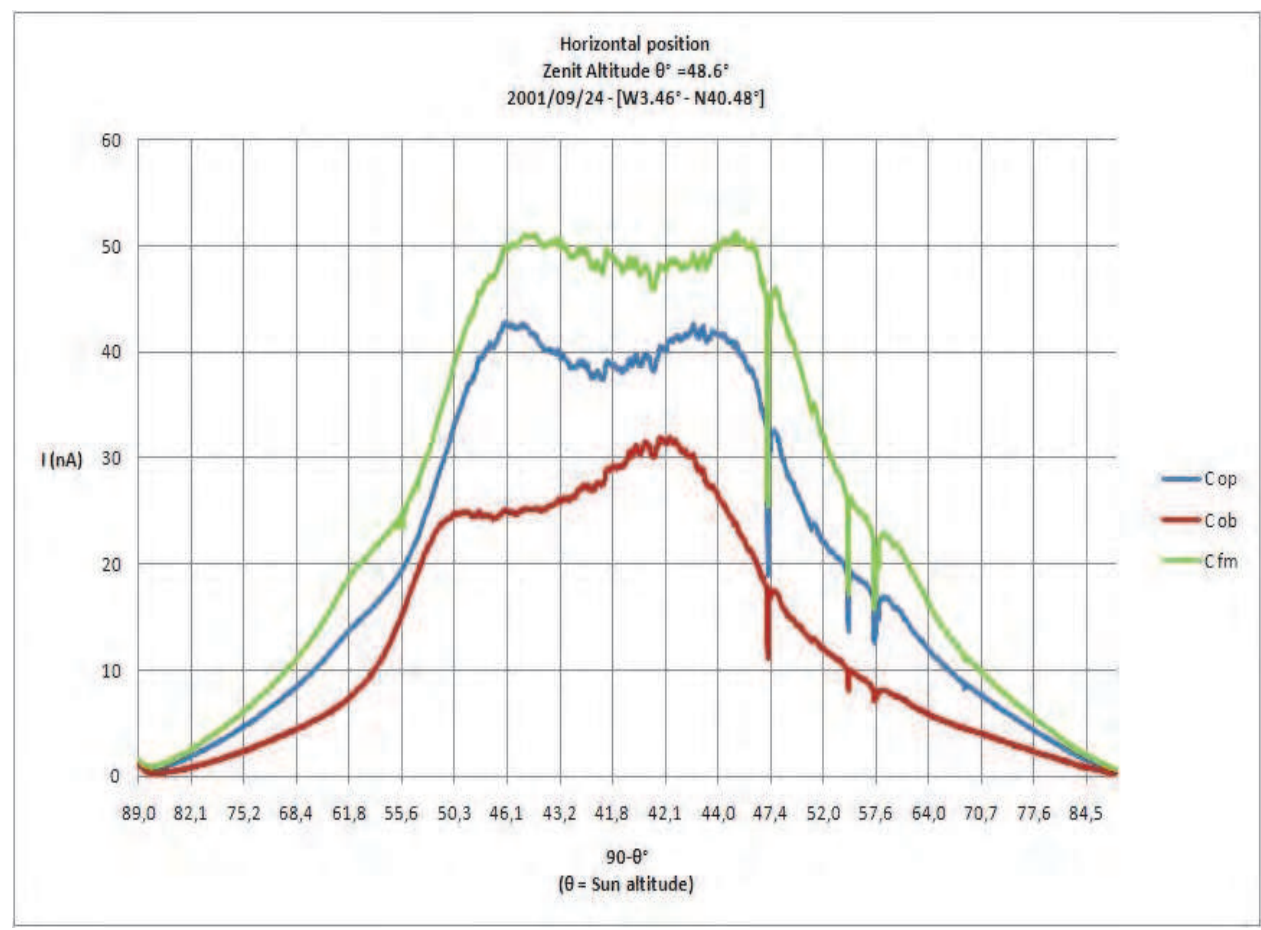

Fig. 12. Comparison of the evolution of the three $C$ photodiodes: flight-model (fm), openblocked (ob) and C open (op) measurement along the day, as the Sun traverses the sky. For this configuration the sun trajectory never enters the nominal FOV. However it is reflected in the inner photodiode walls and two maxima are observed at roughly $45^{\circ}$.

\subsection{Angular characterization of the direct beam: filtered contribution and sunglint}

On the next experiment the platform was inclined and pointing to the maximal solar altitude at $40.2^{\circ}$. Figure 13 shows the current evolution for an $\mathrm{fm}$ photodiode when the sensor platform is oriented towards the Sun maximal altitude position. When the angle of incidence falls between $\pm 30^{\circ}$ with respect to the norm the direct beam is filtered. As was foreseen from laboratory measurements and from the field campaign measurements described in the previous sections, there are two additional maxima at about $\pm 40^{\circ}-45^{\circ}$ angle of incidence. This represents again secondary reflections of the direct Sun beam within the inner housing walls that manage to avoid the filter and produce a current leak. This unfiltered contribution is so big for the $\mathrm{C}$ photodiode that at this stage the total induced current is even bigger than at normal incidence. Beyond that point the signal decays rapidly due to critical angle reflection and the current is induced only by the background sky diffuse irradiance.

In Figure 14 we show for comparison the currents induced in two manipulated C photodiodes. This graph shows that the total irradiance contribution reaching the $\mathrm{C}$ sensing dice is partially filtered and partially non-filtered. We observe that for this channel the non- 
filtered contribution is about $50 \%$ of the total measured signal. Two maxima can be distinguished at $\pm 45^{\circ}$ with respect to the norm. These last two measurements, with the manipulated photodiodes, show an extra non-filtered maximum when the Sun in just above the norm to the sensors which happens when the solar altitude is at $40.2^{\circ}$. In this inclined configuration, this artefact is due to the Sun glint contribution. The direct beam is reflected on the ground and hits the photodiode with an incidence angle of $40.2^{\circ}$, see Figure 15 . In the case of manipulated photodiodes (those that were opened, filled in by normal air, and glued again) this sunglint contribution passes giving a spurious signal. This sunglint contribution seems to be more efficiently rejected in the flight model unit probably due to critical angle issues. The sunglint is not important for REMS application, where the photodiodes will be facing the sky, but may be relevant for other applications with partial view of the ground.

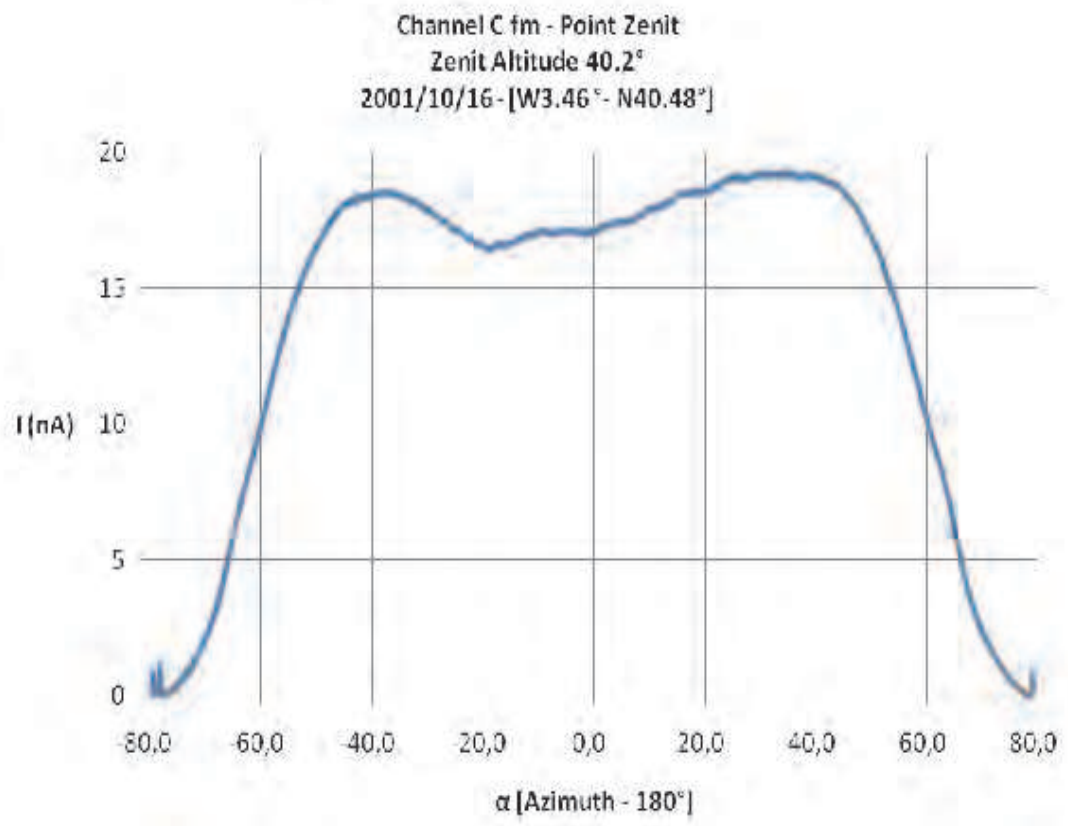

Fig. 13. Evolution of the C fm photodiode measurement along the day, as the Sun traverses the sky crossing the normal to the sensor. The platform was pointing to the maximal altitude position. There are to local maxima at zenithal angles of about $40^{\circ}-45^{\circ}$.

\section{Overall experimental verification and definition of mitigation strategy}

Next we show a summary of this field site verification with all channels measuring simultaneously with an inclined platform configuration, pointing towards the Sun maximal altitude position at $47.9^{\circ}$. In this configuration when the opened photodiodes have been filled in by normal air, the signal shows again an enhanced unfiltered signal due to sunglint. This unfiltered sunglint contribution is clearly distinguished as a central extra peak in channels expecting weak signals (namely $\mathrm{C}$ and $\mathrm{D}$ ). 


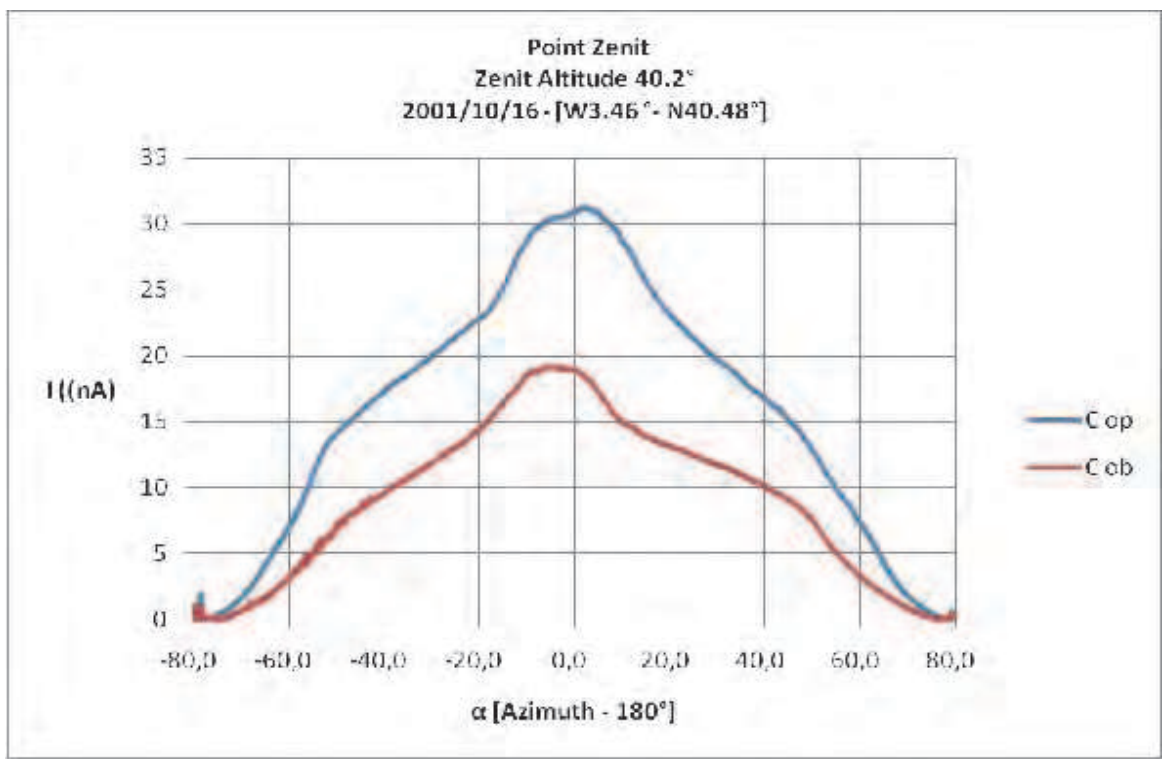

Fig. 14. Evolution of the response of manipulated C photodiodes. Comparison of the openblocked $(o b)$ and $C$ open $(o p)$ measurement along the day, as the Sun traverses the sky. The open-blocked $(o b)$ induced current is the signal induced by unfiltered reflected radiation only whereas the open (op) induced current is the sum of the currents induced on the SiC dice by the unfiltered and filtered radiation.

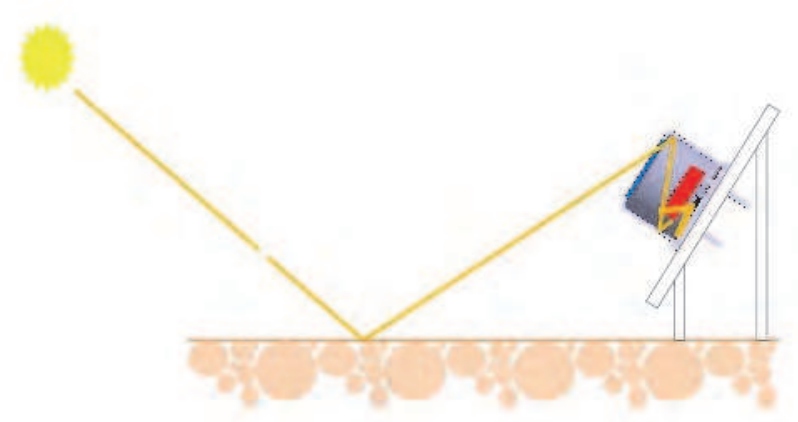

Fig. 15. Sunglint unfiltered contribution for inclined configurations.

For channels A and E the leakage is negligible compared to the nominal signal, see Figure 16. The induced current is smooth and follows the Sun trajectory. The red curve represents the unfiltered contribution (both diffuse and reflected direct irradiance) which in this case is almost negligible. The envelope of these graphs is the sum of the envelope of the background sky diffuse irradiance and, within $\pm 45^{\circ}$, the envelope of direct irradiance.

For channel B there is a significant, and almost constant, background of unfiltered contribution that creates a constant current leakage of about $10 \%$ of the maximal signal, see 
Figure 17. Again, the envelope of these graphs shows one shape for the background sky diffuse irradiance and, within $+/-45^{\circ}$, another envelope for the diffuse plus direct response. The constant background of unfiltered contribution is due to the unfiltered diffuse irradiance of the sky.
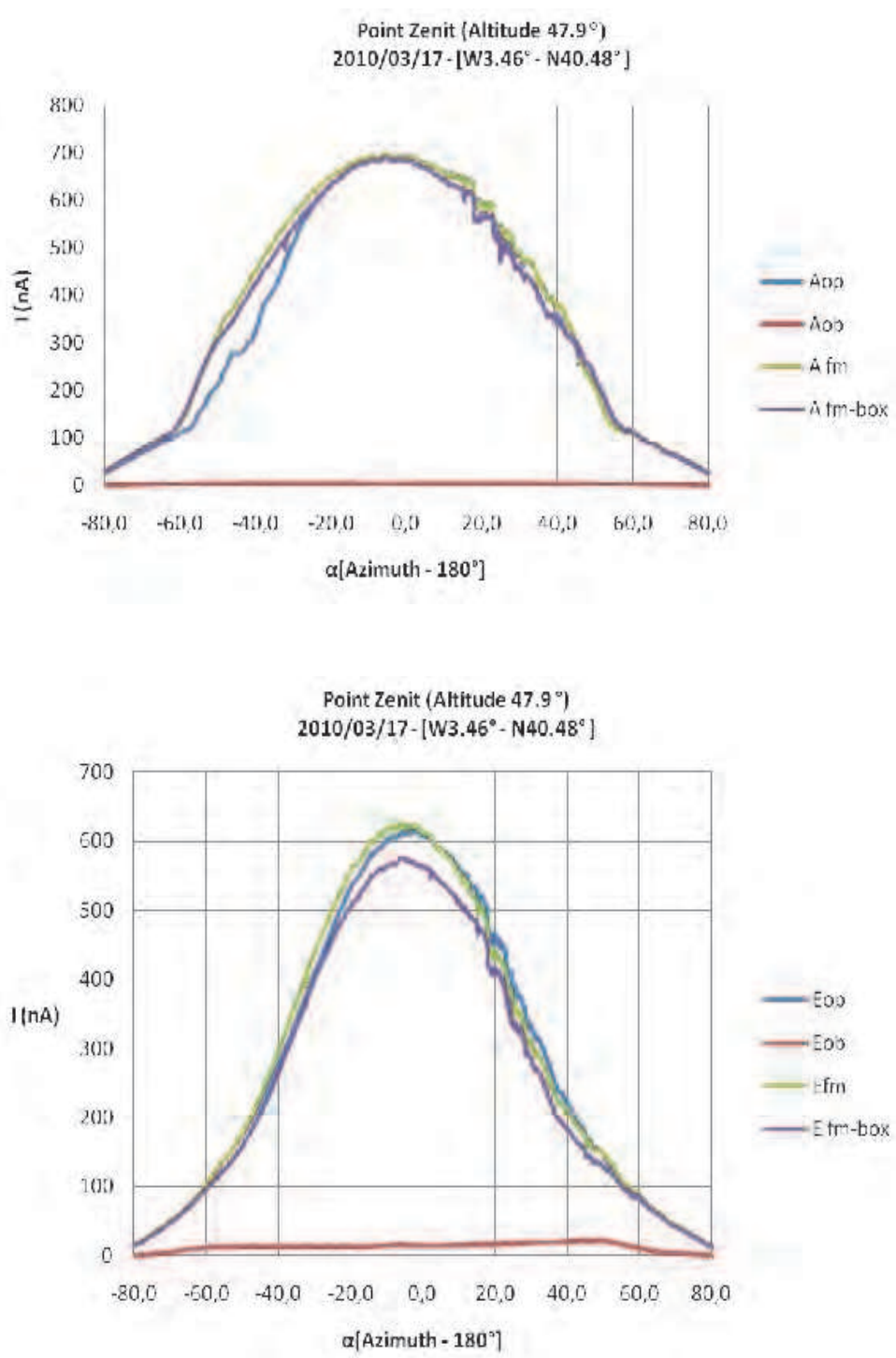

Fig. 16. Diurnal evolution of the measured current for an inclined configuration pointing to the maximal SZA. (Above) Channel A (Below) Channel E. 


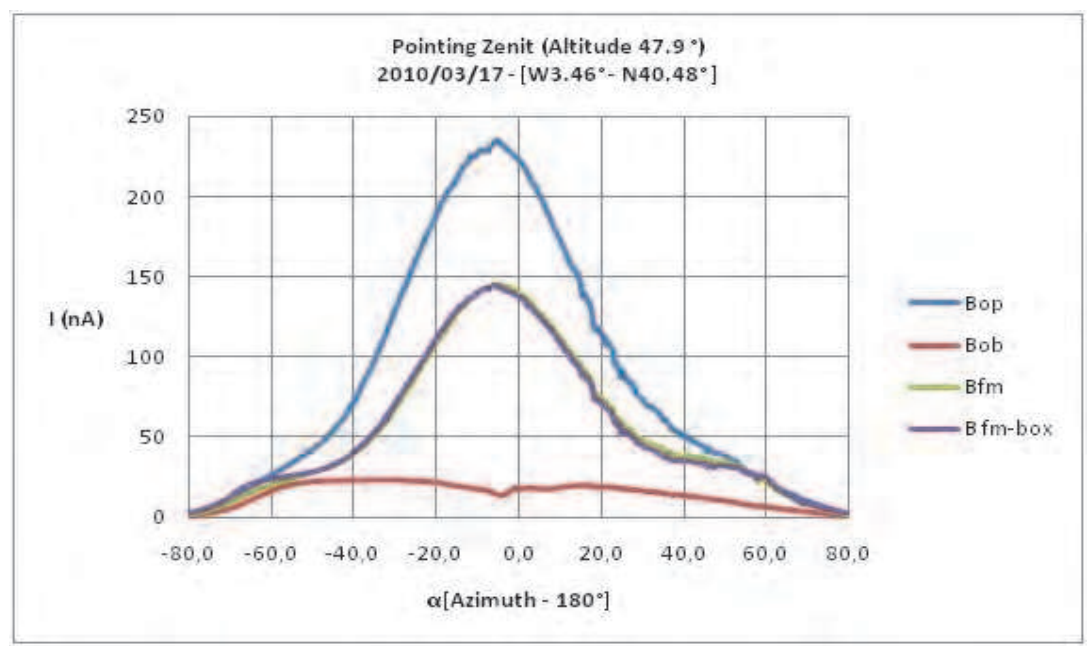

Fig. 17. Diurnal evolution of the B measured current for an inclined configuration pointing to the maximal SZA.

For the C and D manipulated photodiodes, the induced current shows an enhanced, unfiltered response, at SZA of 0 w.r.t to the norm because of sunglint unfiltered reflections, see Figure 17. The flight model units do not show this sun glint contribution. In these ones the two side peaks of direct beam reflections at about $45^{\circ}$ are better seen. Notice that beyond the FOV (beyond $45^{\circ}$ roughly) all the photodiodes show the same response as the ob one, which means that beyond this point the induced current in both $C$ and $D$ photodiodes is pure unfiltered contribution of the background sky diffusive irradiance.

The central enhanced peaks are seen in manipulated photodiodes with weak expected currents, namely C and D channels. And are not seen in the ABC opened, A ob, E ob and B ob. This confirms that is a current leakage of unfiltered contribution of the sunglint contribution of the ground.

Finally by comparing the average response of all photodiodes in the tails (namely beyond $45^{\circ}$ ) of the $A B C$ signal, we conclude again that there is a continuous background unfiltered contribution which is of the order of $2-4 \%$ of the total ( $A B C$ channel) incident UV solar diffuse irradiance, see Figure 19. This $2-4 \%$ introduces a systematic error of current leakage due to diffuse irradiance in all filtered photodiode signals which should be treated as system background error to be subtracted.

In summary, with these observations we can adapt the REMS-UV operational scenario. Namely 1) measure the response of the ABC photodiode when the Sun is out of the FOV (roughly beyond $\pm 45^{\circ}$ ) which is the response to the sky diffuse irradiance alone, and fit its dependence with angle of incidence. 2) Discard those filtered channels whose measurement is comparable with the $2 \%$ of the current induced by diffuse radiation in the ABC channel. 3 ) Subtract this offset contribution to those channels with greater currents, namely, on Earth, A, B and E. 4) Discard measurements performed when the Sun is close to $\pm 45^{\circ}$ with respect to the norm as they may produce unfiltered direct beam contributions due to inner wall reflections. These measurements should be neglected for absolute radiometric measurements (however they could be used for relative comparisons of day to day relative atmospheric changes). 

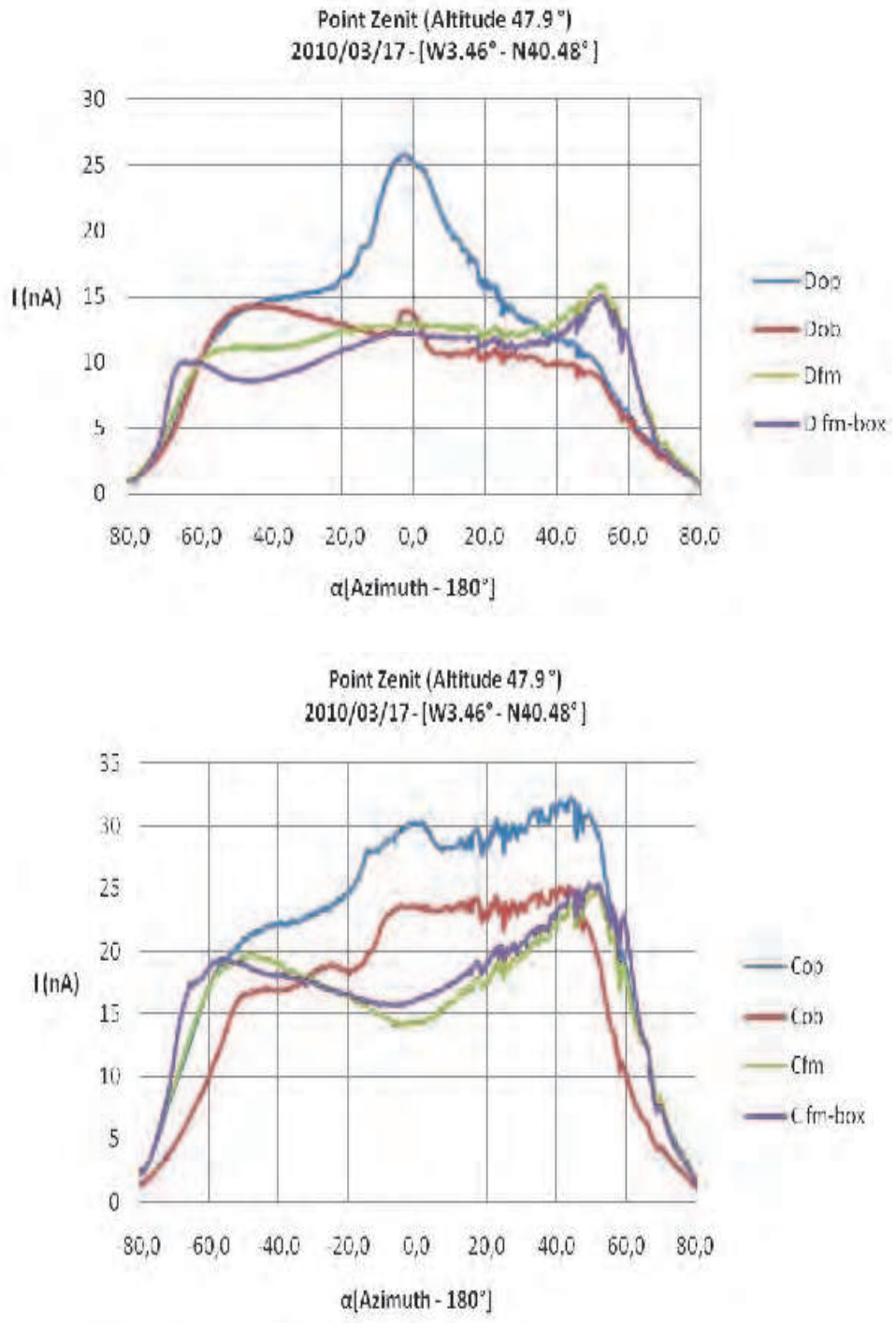

Fig. 18. Diurnal evolution of the measured current for an inclined configuration pointing to the maximal SZA. (Above) Channel D. (Below) Channel C.

Finally regarding REMS measuring strategy during operations on Mars we suggest to define a conservative measuring strategy using the recalibrated currents of photodiodes $A B C, A, B$ and $\mathrm{E}$; and use $\mathrm{C}$ and $\mathrm{D}$ corrected measurements as check points for interpolating algorithms [Zorzano et al. 2009] It must be clarified that on Mars channels C and D are expected to have much greater currents than on Earth since the UVC radiation is not blocked by the atmospheric ozone as on Earth. These measurements have not been performed to date on the Martian surface and thus if during the mission C and D currents 
are much greater than the $2 \%$ of the $\mathrm{ABC}$ diffuse signal these two measurements may be also included as inputs for the interpolating algorithms.

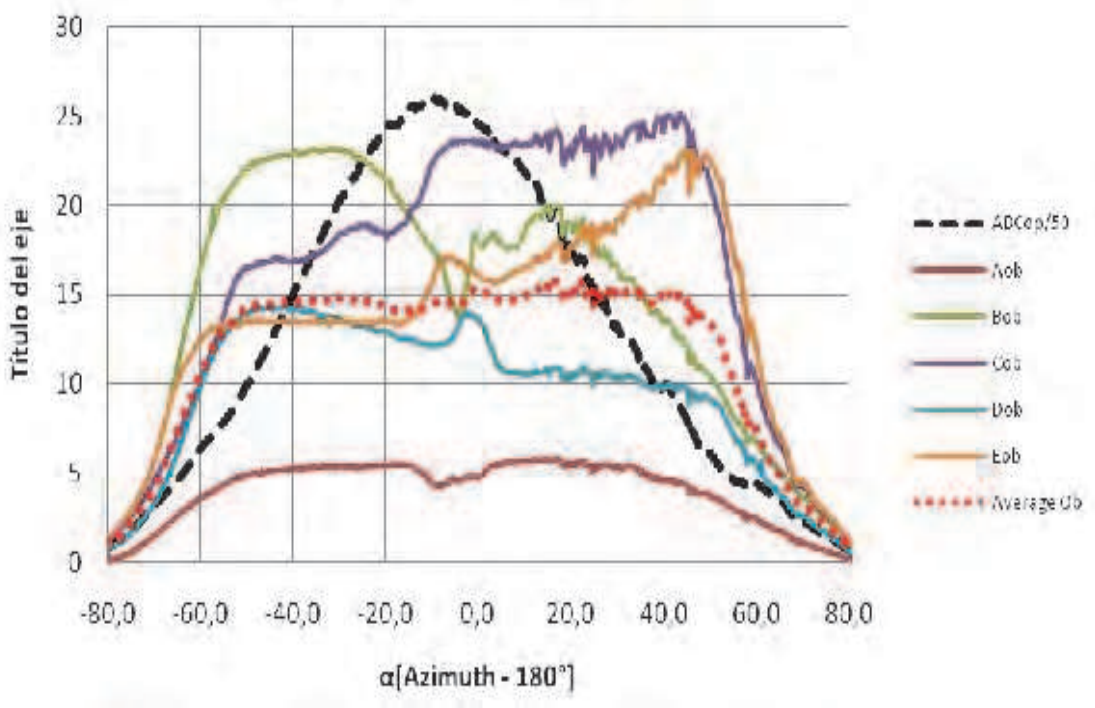

Fig. 19. Evolution along the day of the diffuse unfiltered contribution in filtered channels compared to the total diffuse signal of the total $\mathrm{ABC}$ channel.

\section{Conclusions}

Photodiode sensors are calibrated under controlled laboratory conditions, with collimated, plane light beams at normal incidence with respect to the sensing device. For any other implementation the photodiode needs to be calibrated under representative operation conditions. It is hard to simulate the UV diffuse radiation environment of an atmosphere under laboratory calibration setups. It is also hard to simulate sunglint or secondary reflections induced by reflecting surfaces with small lamps in calibration setups. Dedicated campaigns such as the ones described here should be implemented for this purpose.

In summary, through laboratory and field campaign measurements we have illustrated that:

- The response of the photodiode to a pure, direct, collimated light beam does not decay simply as a cosine law with a cut-off edge at the end of the FOV.

- The sensing dice is also excited by direct beams with angles beyond the nominal FOV that are reflected in the inner housing walls. A second maximal response may be expected for incident angles close to $45^{\circ}$, when the direct beam is reflected on the inner housing walls.

- For certain configurations, also sunglint contributions (or in general reflections of the direct beam source on nearby surfaces) may create unwanted reflected contributions that should taken into account. 
- Equivalently, also the rays of diffuse irradiance with angles greater than the nominal FOV can hit the inner housing walls and reach the sensing dice.

- For the photodiodes used in this test, photon rays with incident angle beyond the nominal FOV, that are reflected in the inner walls of the housing (both the direct and diffuse beam contribution) may avoid the filter action.

- The fraction of radiance that gets to the dice avoiding the filter generates an unexpected unfiltered contribution to the photodiode current. This spurious current leak may be comparable to the nominal contribution, in narrow filtered channels or in spectral ranges where little incident radiation is expected. A mitigation strategy should be implemented to cope with this.

This problem may be common to other sensors used in radiometry or remote sensing applications. Under realistic operation conditions, the radiance comes from multiple paths and it is furthermore not isotropic and also non-normal. Some examples of this situations are:

- If part of the incident radiation hits a surface, a fraction of it may be reflected in an almost specular way and another fraction be scattered away. These other components of irradiance may reach the detector through secondary pathways (example satellite observation of ground reflectance contaminated with sun glint and diffused contribution from lambertian surfaces with different angle of incidence).

- If the medium between the source and detector include scattering agents (such as molecules or aerosols) the diffuse component of the radiation may become significant and therefore reach the sensor from other incident angles.

- Finally even in the case when the direct source beam points to the sensor from angles of incidence beyond its nominal FOV, secondary reflections within the detector housing may allow this radiation to reach the sensing device.

An alternative approach to the use of filters is the use of new tailor-made substrates where the band gap of the photo absorption layer is tuned to select the desired specific range of UV radiation. In particular, recently there is great interest in the development of the so-called solar-blind detectors for applications on Earth. As mentioned above, bellow $280 \mathrm{~nm}$ the UV radiation is absorbed by the terrestrial atmosphere and thus the highest-energy UV light photons from the sun cannot reach the surface. As a result, this region of the solar electromagnetic spectrum constitutes a 'black background' that can be used to detect and control artificial UVC-emitting sources such as flames (and thus detect for instance fires, or missiles). These measurements require detectors that are 'solar blind,' i.e., insensitive to light above $280 \mathrm{~nm}$, and that focus their spectral responsivity in the range of UVC. Current research on solar-blind photodetectors substrates include the development of new substrates such as nano-wires [Delaunay, 2011 and references herein] or $\mathrm{Al}_{x} \mathrm{Ga}_{1-\mathrm{x}} \mathrm{N}$-based substrates [Razeghi, 2002]. This alternative is generally very costful -since it requires development and testing of new materials-, and is not always possible for any arbitrary spectral range. Furthermore the use of new sensing substrates may not be convenient for space applications where all the materials must be qualified for space and the maturity of the technology needs to be well proven.

\section{Acknowledgements}

We thank the support from the REMS team and MSL mission to Mars. This project is funded by the Industry Ministry (CDTI), Science and Innovation Ministry (project ESP2007-65862) 
and Defence Ministry of Spain. We also acknowledge the work of J. Barbero from ALTER Technology Group Spain, Roser Urquí from REMS project, and the National Physical Laboratory (UK) who have contributed to the calibration process.

\section{References}

Cockell, C.S., Catling, D.C., Davis, W.L., Snook, K., Kepner, R.L., Lee, P., McKay, C.P., 2000. The ultraviolet environment of Mars: biological implications. Past, present, and future. Icarus 146, 343.

Córdoba-Jabonero, C., Lara, L.M., Mancho, A. M., Marquez, A., Rodrigo, R., (2003). Solar Ultraviolet transfer in Martian atmosphere: biological and geological implications Planet. Space Sci. 51, 399-410.

Cordoba-Jabonero, C., Zorzano, M.-P., Selsis, F., Patel, M.R., Cockell, C.S., (2005). Radiative habitable zones in Martian polar environments. Icarus 175, 360-371.

Delaunay J-J, Li, Y., Tokizono, T., Liao, M., Koide, Y. (2011). Wide-bandgap nanowires for UV-light detection. Optoelectronics \& Communications. SPIE. $10.1117 / 2.1201102 .003466$

Gómez-Elvira, J., and REMS Team, Environmental monitoring station for Mars Science Laboratory, Proceedings of LPI Contributions, 1447, p. 9052. (2008).

Holland, H. D., 1978. The Chemistry of the Atmosphere and Oceans. Wiley Interscience, New York.

Kinch, K.M., Merrison, J.P., Gunnlaugsson, H.P., Bertelsen, P., Madsen, M. B., Nørnberg, P. (2006). Preliminary analysis of the MER magnetic properties experiment using a computational fluid dynamics model. Planet. Space. Sci. 54, 28-44.

Mateshvili, N., Fussen, D., Vanhellemont, F., Bingen, C., Dodion, J., Muller, C., Depiesse, C., Perrier, S., Bertaux, J.L., Dimarellis, E. 2006. Martian clouds distribution obtained from SPICAM nadir UV measurements: preliminary results. Second workshop on Mars atmosphere modelling and observations, held February 27 - March 3, 2006 Granada, Spain. Edited by F. Forget, M.A. Lopez-Valverde, M.C. Desjean, J.P. Huot, F. Lefevre, S. Lebonnois, S.R. Lewis, E. Millour, P.L. Read and R.J. Wilson. Publisher: LMD, IAA, AOPP, CNES, ESA.

Montmessin, F., Quemerais,E., Bertaux,J. L. , Korablev,O. , Rannou,P. And Lebonnois,S. (2006). Stellar occultations at UV wavelengths by the SPICAM instrument: Retrieval and analysis of Martian haze profiles, J. Geophys. Res., 111,No. E12, E12S06

Mukhin, L.M., Koscheev, A.P., Dikov, Y.P., Hurth, J., WRanke, H., (1996). Experimental simulations of the photodecomposition of carbonates and sulphates on Mars. Nature 379, 141.

Patel, M.R.; Zarnecki, J.C. and Catling, D.C. (2002). Ultraviolet radiation on the surface of Mars and the Beagle 2 UV sensor. Planetary and Space Science, 50(9), pp. 915-927.

Patel, M.R., Bérces,A., Kolb, C., Rettberg, P., Zarnecki, J. C. and Selsis, F., (2003). Seasonal and Diurnal Variations in Martian Surface UV Irradiation: Biological and Chemical Implications for the Martian Regolith. International Journal of Astrobiology, 2 (1), pp 21-34.Quin, R.C., Zent, A.P., McKay, C.P., (2001). Photodecomposition of carbonates on Mars. Lunar Planet. Sci. Conf. XXXII, 1463. 
Patel, M.R., Bérces, A., Kerkgyrt, T., Ront, G., Lammer, H. and Zarnecki, J.C., 2004. Annual Solar UV Exposure and Biologically Effective Dose Rates on the Martian Surface. Advances in Space Research, 33 (8), pp 1247-1252.

Razeghi, M. Short-wavelength solar-blind detectors-status, prospects, and markets. Proceedings of the IEEE, Jun 2002, vol 90., 6, pp. 1006-1014.

Rodrigo, R., Garcia-Alvarez, E., Lopez-Gonzalez, M. J., Lopez-Moreno, J., (1990). A nonsteady one-dimensional theoretical model of Mars' neutral atmospheric composition between 30 and 200 KM. Journal of Geophysical Research (ISSN 01480227), vol. 95 , Aug. 30 p. $14795-14810$.

Vazquez L, Zorzano MP, Jimenez S. (2007). Spectral information retrieval from integrated broadband photodiode Martian ultraviolet measurements. Optic Letters 32(17) 25962598.

Zorzano, M.P., Vázquez, L., Jiménez, S. (2009). Retrieval of ultraviolet spectral irradiance from filtered photodiode measurements. Inverse Problems, vol. 25 pp.115023-115032 (2009).

Zorzano, M.-P., Mancho, A.-M., Vazquez, L. (2005). Numerical integration of the discreteordinate radiative transfer equation in strongly non homogeneous media. Applied Mathematical and Computation, 164, 263-274.

Zorzano M.-P., Cordoba-Jabonero C (2007). Influence of aerosol multiple scattering of ultraviolet radiation on Martian atmospheric sensing. Icarus 2007, vol. 190, no2, pp. 492-503. 


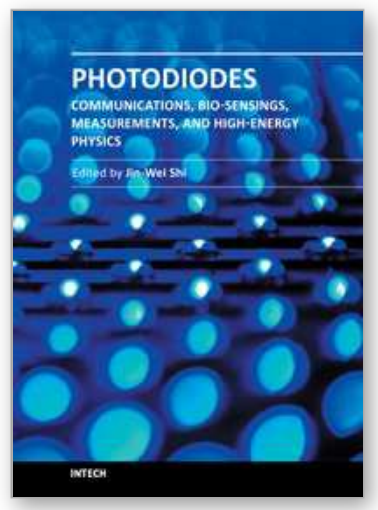

\author{
Photodiodes - Communications, Bio-Sensings, Measurements and \\ High-Energy Physics
}

Edited by Associate Professor Jin-Wei Shi

ISBN 978-953-307-277-7

Hard cover, 284 pages

Publisher InTech

Published online 06, September, 2011

Published in print edition September, 2011

This book describes different kinds of photodiodes for applications in high-speed data communication, biomedical sensing, high-speed measurement, UV-light detection, and high energy physics. The photodiodes discussed are composed of several different semiconductor materials, such as $\mathrm{InP}, \mathrm{SiC}$, and $\mathrm{Si}$, which cover an extremely wide optical wavelength regime ranging from infrared light to X-ray, making the suitable for diversified applications. Several interesting and unique topics were discussed including: the operation of highspeed photodiodes at low-temperature for super-conducting electronics, photodiodes for bio-medical imaging, single photon detection, photodiodes for the applications in nuclear physics, and for UV-light detection.

\title{
How to reference
}

In order to correctly reference this scholarly work, feel free to copy and paste the following:

María-Paz Zorzano, Javier Martin-Soler and Javier Gómez-Elvira (2011). UV Photodiodes Response to NonNormal, Non-Colimated and Diffusive Sources of Irradiance, Photodiodes - Communications, Bio-Sensings, Measurements and High-Energy Physics, Associate Professor Jin-Wei Shi (Ed.), ISBN: 978-953-307-277-7, InTech, Available from: http://www.intechopen.com/books/photodiodes-communications-bio-sensingsmeasurements-and-high-energy-physics/uv-photodiodes-response-to-non-normal-non-colimated-anddiffusive-sources-of-irradiance

\section{INTECH}

open science | open minds

\author{
InTech Europe \\ University Campus STeP Ri \\ Slavka Krautzeka 83/A \\ 51000 Rijeka, Croatia \\ Phone: +385 (51) 770447 \\ Fax: +385 (51) 686166 \\ www.intechopen.com
}

\author{
InTech China \\ Unit 405, Office Block, Hotel Equatorial Shanghai \\ No.65, Yan An Road (West), Shanghai, 200040, China \\ 中国上海市延安西路65号上海国际贵都大饭店办公楼405单元 \\ Phone: +86-21-62489820 \\ Fax: +86-21-62489821
}


(C) 2011 The Author(s). Licensee IntechOpen. This chapter is distributed under the terms of the Creative Commons Attribution-NonCommercialShareAlike-3.0 License, which permits use, distribution and reproduction for non-commercial purposes, provided the original is properly cited and derivative works building on this content are distributed under the same license. 